\title{
Coagulation and fibrinolysis in injured patients
}

\author{
D. INNES AND S. SEVITT
}

From the Pathology Department, Birmingham Accident Hospital

SYNOPSIS Serial changes in coagulation and fibrinolysis studied among 42 patients admitted to hospital with a wide variety of injuries are reported. The first hours after trauma are dominated by an acceleration of fibrinolysis (clot lysis) and clotting time which are often followed by an abrupt rebound to prolonged fibrinolysis and normal clotting. Evidence is presented that acceleration of fibrinolysis is due to flooding of the circulation by plasminogen activator and that prolongation is probably due to an inhibitor. A prolonged prothrombin time, increased prothrombin consumption index, an acceleration of the heparin-retarded clotting time, and a fall in the platelet count are also frequent during the first hours after injury. There is evidence also of an early deficiency in factor $\mathrm{V}$ and the onset of a fall in factor VII and prothrombin.

The following days are characterized by continued prolongation of fibrinolysis, a lengthening of clotting time, and an increased prothrombin consumption index suggestive of a defect in thromboplastin generation. Subsequent periods of prolonged fibrinolysis may develop. Prothrombin time often continues prolonged for one to three weeks and may vary phasically; plasma prothrombin and factor VII are reduced but there is now little change in factor V. The platelet count continues to fall for one to three days, then a thrombocytosis develops, often with abnormally high platelet levels, a week or so later. Plasma fibrinogen rises within 24 hours to reach a plateau maximum a few days later and levels remain high for prolonged periods in the severely injured. Various changes are related to or influenced by the severity of trauma. Mechanisms are discussed, including thrombosis in vivo, and reference is made to homeostatic significance and its possible breakdown.

This paper is concerned with serial coagulation and fibrinolytic changes from soon after injury until recovery or death. The subject, of basic physiological interest, has assumed a possible importance since the postulate that 'irreversible shock' after blood loss owes its origin to an hypercoagulable state (Crowell and Read, 1955; Turpini and Stefanini, 1959; Hardaway, 1962).

Quickened blood clotting after experimental haemorrhage was first studied by Cannon and his colleagues (Cannon and Gray, 1914; Gray and Lunt, 1914; Cannon and Mendenhall, 1914) but it was first recognized by William Hewson (1772) (see Gulliver, 1846). He observed that 'the blood taken later ... coagulated in less and less time, till . . . the blood which issued last coagulated first'. Quickened clot lysis after surgery was first found by Macfarlane and Biggs (1946) and in a few cases of trauma and burns by Tagnon, Levenson, Davidson, and Taylor (1946) and Cliffton (1952); but the fluid, incoagulable state of post-mortem blood after sudden death has also been known since the eighteenth cen-

Received for publication 15 August 1963. tury. Hewson (1772) quotes a Dr. Davy and M. de Senai (1749) and Mole (1948) quotes Morgani (1769) and Hunter (1794). Rapid lysis of post-mortem clots by a fibrinolytic agent is responsible (Mole, 1948).

Recent studies in man after injury or operation have been confined to coagulation phenomena (Warren, Amdur, Belko, and Baker, 1950; Scott and Crosby, 1954) but observations on coagulation and fibrinolysis have been made in animals during the shock phase (Turpini and Stefanini, 1959; Bergentz and Nilsson, 1961).

\section{OUTLINE OF THE INVESTIGATIONS}

Altogether 42 in-patients ( 35 males) with various injuries, including one burned patient $(14 \%$ body area), were studied. Twenty-two men and one woman were 16 to 40 years old; seven men and six women were older than 50 years. Some were discharged within a few days but most were in hospital for weeks. Seven with severe trauma died: two within a few hours, one at one and half days after the accident, two on the seventh and ninth days and 
the other two after one and four months respectively. Severity of injury has been broadly divided on clinical grounds into 'moderate' and 'severe' trauma. Moderate injuries (22 patients) include fractures of a long bone, ankle, wrist, or patella; a minor head injury (short unconsciousness); lacerations and certain combinations not amounting to serious injury or producing much blood loss. Severe injuries (20 cases) are mainly fractures of two or more long bones; pelvic fractures with other fractures or injuries; serious head injuries; severe abdominal or thoracic trauma; and various multiple injuries combining in severity and/or blood loss.

Some patients were studied repeatedly by a battery of tests from soon after injury until discharge or death and others less intensively or for shorter periods. Because many early changes are shortlived, the first few hours after injury were investigated by venous sampling at half to one-hourly intervals in subjects admitted soon after the accident. At this time the severely injured were under close surveillance in the major injuries unit of the hospital. Frequent clinical observations, including pulse and blood pressure, were made; blood volume was estimated as required and treatment, including blood transfusion and surgery, was carried out by the clinical staff. Subsequent blood samples were taken between $10.0 \mathrm{a} . \mathrm{m}$. and 12 noon on account of the diurnal variation in fibrinolytic activity reported by Fearnley, Balmforth, and Fearnley (1957). Daily tests for 10 days and thereafter less frequently were done on many patients. Five patients received oral anticoagulant therapy but, with special exceptions, the data after its initiation are excluded from this paper.

\section{METHODS}

Venous blood was drawn into siliconed syringes without venous stasis except in some shocked cases when it was unavoidable. The sample was divided into polystyrene tubes for estimating clotting time, heparin-retarded clotting time, prothrombin time, and Thrombotest, and into glass tubes for fibrinolysis estimation and other tests. All tests were done in duplicate.

FIBRINOLYSIS TIME The clot-lysis method (Fearnley et al., 1957) using citrated blood standing in iced-water up to testing (less than one hour from collection) was modified by reducing the blood concentration from $10 \%$ to $5 \%$ so shortening normal lysis time, the final thrombin concentration to 0.5 unit per $\mathrm{ml}$. so minimizing contaminating plasma, and the final volume of diluted blood to $2 \mathrm{ml}$. The incubating clots were observed frequently up to 10 hours and less frequently up to 24 hours. The end-point of lysis was virtual disappearance of the clot and the time from incubation at $37^{\circ} \mathrm{C}$. was the fibrinolysis time (lysis time). Lysis times ranged between three and 10 hours in $87 \%$ of 94 tests on 51 normal subjects between
16 and 48 years of age. Variation in duplicate tests was less than $\pm 10 \%$ of the mean in $91 \%$ of estimations.

STREPTOKINASE-ACTIVATED FIBRINOLYSIS TIME To obtain an index of plasminogen, clot lysis was accelerated by a standard amount of streptokinase (Dornokinase, Burroughs and Wellcome, a mixture of streptokinase and streptodornase). Streptokinase was added (to give a final dilution of 20 units per $\mathrm{ml}$.) after clotting the diluted blood with thrombin and just before placing the tube in the water bath. The amount was chosen after pilot trials. Activated clot lysis time was between one and two and a half hours in $95 \%$ of 80 tests done on 47 normal subjects, the variations no doubt reflecting streptococcal antibody in plasma. Variation in duplicate tests was less than $\pm 10 \%$ of the mean in $83 \%$ of tests. This test and the 'spontaneous' lysis time were done simultaneously.

WHOLE BLOOD CLOTTING TIME One millilitre of blood was transferred from the syringe to a polystyrene tube $\left(8 \mathrm{~mm}\right.$. diameter) at $37^{\circ} \mathrm{C}$. The end-point was firm coagulation, tested for by gentle tilting. Variations of duplicate tests were within $\pm 9 \%$ of the mean. Ninety-one per cent of 68 tests on 43 control subjects were between nine and $16 \frac{1}{2}$ minutes.

HEPARIN-RETARDED CLOTTING TIME Poller's (1954) technique for plasma was slightly modified to ensure a standard normal range (six to 11 minutes) because of variations in different batches of heparin of the same labelled strength. The activity of a fresh batch of heparin on normal plasma was always compared with the current one by a calibration curve. Ninety-three per cent of 87 tests on 55 normal subjects were within the range six to 11 minutes.

PROTHROMBIN-CONSUMPTION INDEX (RESIDUAL SERUM PROTHROMBIN) Using the technique of Biggs and Macfarlane (1953) this was less than $15 \%$ of plasma prothrombin in 33 of 37 tests in 26 normal subjects. Results in excess of $15 \%$ are considered abnormal for the present purpose.

ONE-STAGE PROTHROMBIN TIME The thromboplastin was an extract of acetone-dried human brain. The plasma was recalcified with calcium chloride, M/40. Results (normal 11 to 12 seconds) longer than 13 seconds were abnormai.

THROMBOTEST Tests were done according to the makers' instructions for plasma except that $3.8 \%$ citrate was the anticoagulant. This gives the same results as the lower concentration recommended. The reagent was checked daily with two dilutions of normal plasma against the calibration curve supplied.

PLASMA PROTHROMBIN ASSAY AND THE TWO-STAGE PROTHROMBIN TEST Prothrombin was assayed by the twostage area technique of Biggs and Douglas (1953) slightly modified. Results varied from $80 \%$ to $138 \%$ of the mean in 12 of 13 assays in seven normal subjects (18 to 29 years). Prothrombin by the two-stage method at one minute's incubation gave an excellent correlation: results were 
between $83 \%$ and $115 \%$ of the mean in 14 of the 15 tests in nine normal subjects.

PLASMA FIBRINOGEN Using Watson's (1961) precipitation method turbidities were read spectrophotometrically at $420 \mathrm{~m} \mu$. The readings were translated to absolute values by a calibration curve with fibrinogen determined gravimetrically. The added citrate was not corrected for.

BLOOD PLATELETS These were counted by the technique of Brecher and Cronkite (1950).

BLOOD EOSINOPHILS A direct wet-field method employing eosin-acetone as a diluent was used.

Other tests are referred to in the text.

\section{RESULTS}

EARLY CHANGES IN FIBRINOLYSIS AND CLOTTING TIME Altogether 28 patients were studied during this phase, 15 serially. Typically, fibrinolysis and clotting became quickened soon after injury but within a few hours they returned to normal or became prolonged. Figure 1 shows lysis time accelerated down to one and a quarter hours and clotting time to six and a half minutes between two and a half and three and a half hours after injury in a patient with fractures of the pelvis and femora; lysis time then quickly passed through the normal range and was greatly prolonged by six hours after injury whilst clotting time became normal. Results in six other patients are shown in Fig. 2 and for two others in Figures 5 and 8. Quickened lysis was
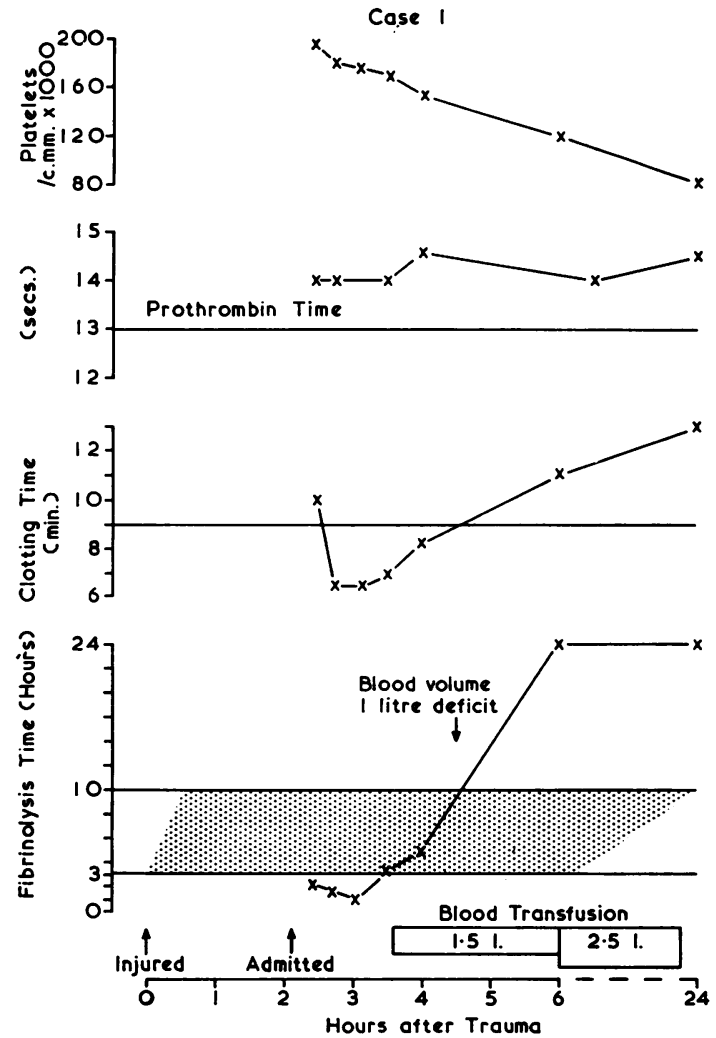

FIG. 1. Serial changes in case 1 .
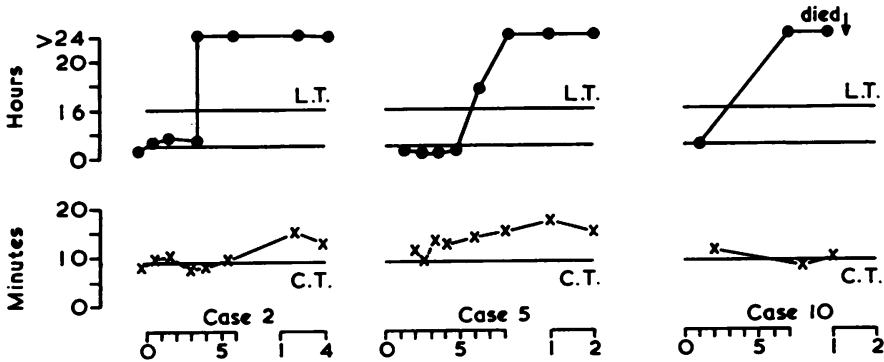

인 $\begin{array}{rr}24 \\ \vdots\end{array}$
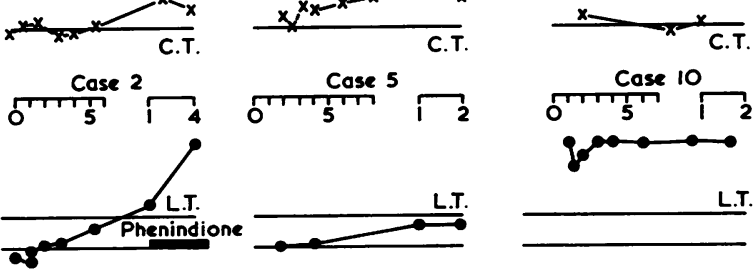

FIG. 2. Early changes in lysis time (L.T.) and clotting time (C.T.) in six patients.
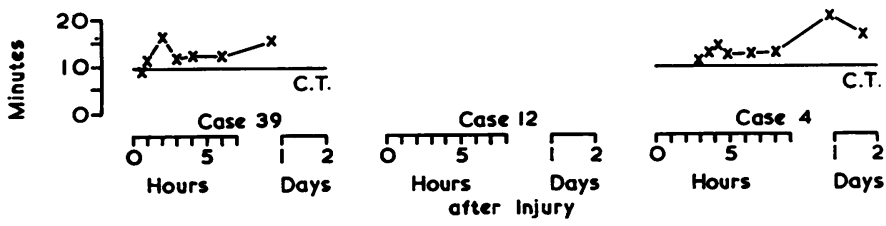
found in 11 of the 15 subjects studied serially. Usually it is quickly followed by prolongation of lysis (cases 2 and 5) and this included one patient (case 10) who died next day. Occasionally it is followed by normal lysis during this period (cases 12 and 39, Fig. 2; and case 3, Fig. 8). Sometimes the first and subsequent tests show prolonged lysis ( $>24$ hours) as in case 4 ; but then the early acceleration may have been missed. Indeed detection of the early accelerated phase depends on the timing of the venepunctures and it could be readily overlooked if the first of a series of samples were obtained more than three to five hours after injury. Our earliest samples were between three quarters and one hour after trauma (three cases) and lysis was already fast ( $<$ three quarters of an hour to one and three quarter hours). The process of post-traumatic quickening was not observed so that it must occur very soon after injury. Assuming it begins within minutes of injury its duration was estimated as one to two hours in two patients, two to three hours in four, and three to five hours in three subjects. Severity of injury probably influences this, because the first two were moderately and the last three severely injured. The shortest lysis times (<one half hour to three quarters of an hour) were found within an hour of injury in two patients surviving the early period and shortly before death in two who died at two and three and a half hours after trauma. These lysis times probably represent considerable concentrations of plasminogen-activator (vide infra) because by tests in vitro very large amounts of the activator urokinase are needed to speed normal clot lysis to say two hours or one hour (Fig. 3). For example, in the top line, clot lysis was quickened from seven to four hours by only 5 units of urokinase per millilitre in the test but 50 to 150 units were needed to speed it to two and a third and one and a half hours respectively. These values correspond to $100,1,000$, and

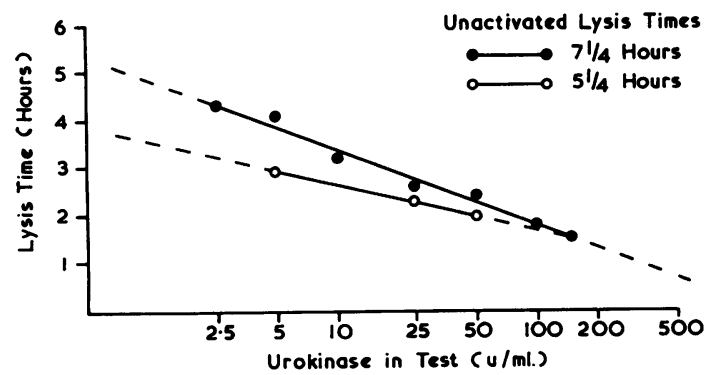

FIG. 3. Clot lysis times of two samples of normal blood after adding various amounts of urokinase to the test. The lysis times without urokinase were seven and a quarter hours (upper line) and five and a quarter hours (lower line). Concentrations of urokinase are plotted logarithmetically.
3,000 units urokinase per millilitre of blood respectively.

The onset of accelerated clotting, that is, normal results preceding shortened clotting, was found in a few patients. Usually it began later than quick lysis and was of shorter duration but this was not invariable and in some subjects the quick clotting phase corresponded to the onset of prolonged fibrinolysis. Accelerated clotting times were usually between seven and nine minutes and the shortest was five minutes (case 3, Fig. 8).

ASSOCIATED CHANGES DURING THE FIRST FEW HOURS Other early changes were the onset of a fall in the platelet count; commonly a moderate prolongation of prothrombin time and low Thrombotest activity; significant increases in prothrombin consumption index; and transient shortening of the heparinretarded clotting time. These will be considered later.

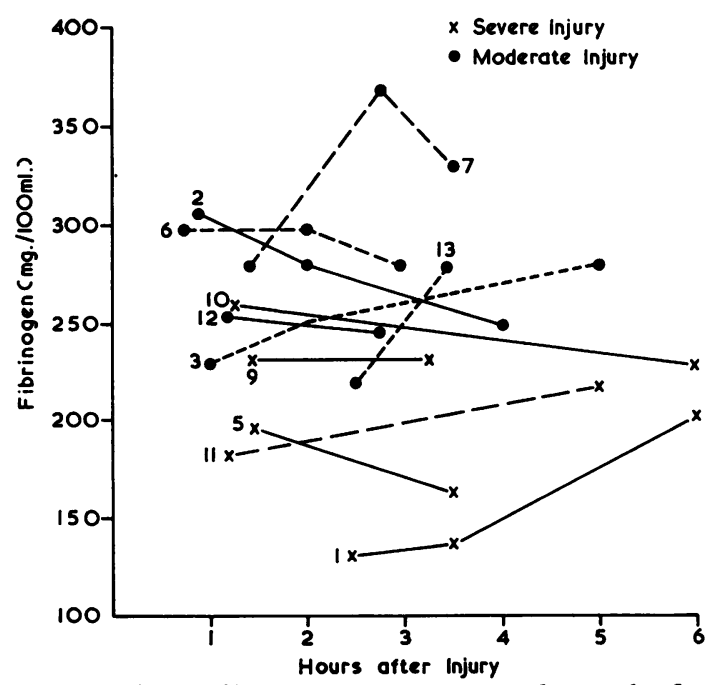

FIG. 4. Plasma fibrinogen concentrations during the first few hours after injury. Numerals are case numbers. There is no consistent or significant pattern of changes at this time although the values in some cases are low.

Fibrinogen estimations in 12 subjects showed some variations at this time (Fig. 4) but except for some low or low-normal results, they seemed without pattern and were also unrelated to blood transfusion. Evidence of fibrinogenolysis in plasma was sought for in early samples from seven patients; the lysis time was quickened in six; the fibrinogen concentrations of the plasma to which the fibrinolytic inhibitor epsilon-amino-caproic acid (E.A.C.A.) $\left(10^{-2} \mathrm{M}\right)$ had been added was the same as in the sample without the inhibitor. This indicates that the quick clot lysis in vitro is not accompanied by significant action in vivo at least on fibrinogen. 
LATER CHANGES IN FIBRINOLYSIS Prolongation of lysis time was the dominant feature from a few hours after injury. Figure 1 shows its relatively sudden onset five to six hours after injury and further examples are seen in Figure 2 (cases 2, 5, and 10). Figure 5 shows the period of prolonged lysis in a man with badly fractured ankles and an injured spine (case 9); lysis time was prolonged until about the fifth day when it returned nearly to normal. Prolongation of clot lysis was usual; it was found in all the 14 severely injured subjects studied and in 12 of the 19 moderately injured. The period varied considerably: it ranged from two or three days in a few subjects to 26 days in the burned patient but was usually between four and 11 days. The duration was unrelated to the simultaneous rise in plasma fibrinogen level.

Second periods of prolonged lysis were often observed (Fig. 5) and occasionally third periods were noted. Allowing for deaths, early discharges, and infrequent tests, a second phase was found in nine out of 11 severely injured compared with one out of four with moderate injuries. An acute clinical event-surgery, fracture manipulation, pulmonary embolism, or infection-usually accompanied or
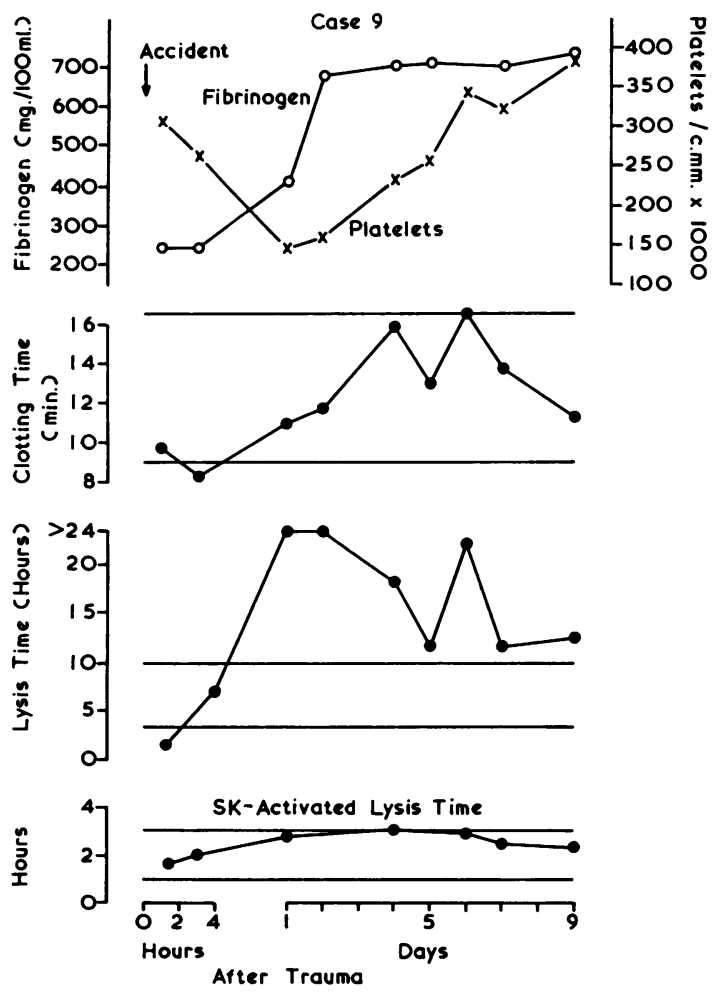

FIG. 5. Serial changes in case 9. shortly preceded the onset of the second or third periods. In one patient (case 27) lysis time previously prolonged was normal on the fifth day shortly after the patient had collapsed; but next day and subsequently lysis times were prolonged; the patient died on the ninth day and necropsy showed major pulmonary embolism. Late onset of prolonged lysis was found in two subjects in whom the early acceleration had been followed by normal lysis times. This is seen in Fig. 8 where prolongation of lysis developed during an attack of fat embolism.

Second, third, and late periods of prolonged lysis are probably similar in origin to the first period in that they follow an acute 'stressing' event. They are also possibly preceded by transient quickening of lysis which may have been missed in many subjects as the tests were not frequent enough at this time. However in case 32 lysis time was normal before a femur was nailed and was greatly prolonged at three quarters of an hour after surgery. It is of interest that the spleen had been removed on the day of injury.

ACTIVATOR AND INHIBITOR OF FIBRINOLYSIS Quick clot lysis early after injury depends technically on cooling the blood in iced water during the interval before the test is set up. Lysis time lengthens greatly if the blood is allowed to stand at room temperature for a time. This lability is similar to that found by Fearnley, Revill, and Tweed (1952) for normal clot lysis and suggests that the same labile activator is responsible. The subsequent slowing of clot lysis suggests either that an inhibitor appears or that activator is 'exhausted'. Evidence of activator and inhibitor was found by additional experiments in which serial lysis times of the patient's blood, a normal sample, and the latter after adding $10 \%$ or $20 \%$ of the patient's blood, were compared. For comparison the lysis time of the mixed specimen is expressed as a percentage acceleration or prolongation of that of the normal sample (see footnote to Fig. 6). Figure 6 shows that $20 \%$ of the patient's blood taken at one and a quarter, one and a half, and two hours after injury when clot lysis was quickened, accelerated the lysis time of a normal sample by $75 \%, 65 \%$, and $33 \%$ respectively; whilst that taken next day when clot lysis was slowed, prolonged normal lysis by $33 \%$. Figure 7 summarizes the results in 12 patients: blood with quickened lysis always accelerated that of normal blood well beyond the dilution effect and blood with prolonged lysis always prolonged the normal. ABO compatibility or incompatibility did not influence the results.

Serial streptokinase-activated lysis times indicated that prolonged lysis was not due to deficiency of 

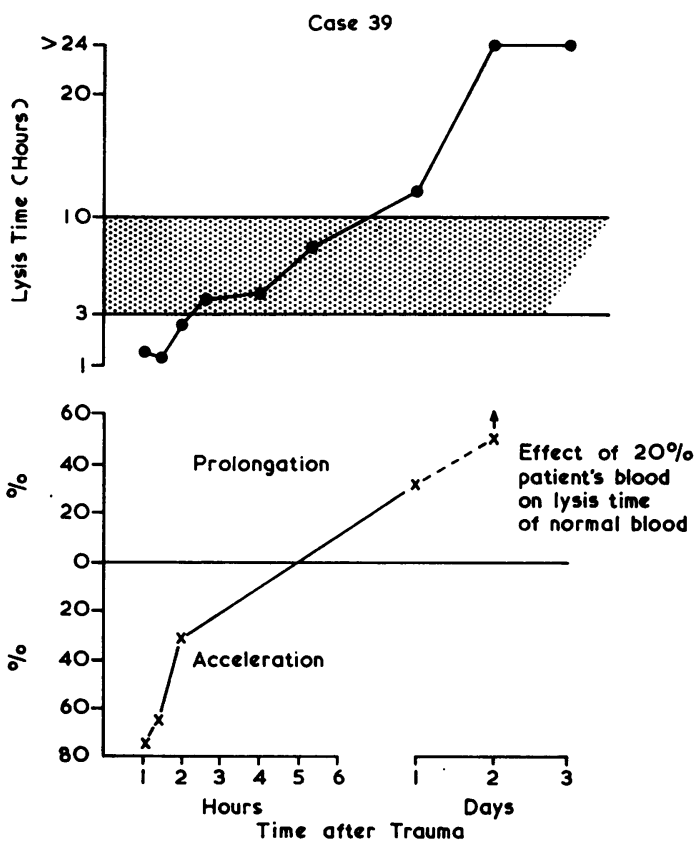

FIG. 6. Fibrinolysis changes in case 39 (upper curve) are compared with the effects on the lysis time of normal blood of adding $20 \%$ of the patient's blood (lower curve). Lysis time of the normal is accelerated or prolonged according to acceleration or prolongation of the patient's lysis time. Percentage acceleration of lysis time $=$

$$
\frac{L T \text { normal }-L T \text { mixed specimen }}{L T \text { normal }-L T \text { patient }} \times 100
$$

and percentage inhibition $=$

$$
\frac{L T \text { mixed specimen }-L T \text { normal }}{L T \text { normal }} \times 100
$$

$L T=$ fibrinolysis time. In the formula for percentage inhibition the value for the patient's blood was not taken into account because many values were longer than 24 hours.

plasminogen and unlikely to result from exhaustion of activator. An example (Fig. 5) shows that the activated lysis times were within the quickened normal range of streptokinase activation.

Lysis times after adding E.A.C.A. were estimated on blood taken within two hours of injury in five patients. The E.A.C.A. was added before the diluted blood was clotted so as to permit inhibitor action before plasminogen-activation. With a final concentration of $\mathbf{M ~ 0 . 5} \times 10^{-5}$ E.A.C.A., lysis times were prolonged by $17 \%$ to $40 \%$ (mean $20 \%$ ) compared with $20 \%$ to $60 \%$ prolongation (mean $35 \%$ ) in 11 normal blood samples. Higher concentrations had a more inhibitory effect and $0.5 \times 10^{-3} \mathrm{M}$. produced lysis times $>24$ hours in normal and patient's samples. The concentrations of E.A.C.A. were much less than that needed to inhibit plasmin and were of the order which inhibit plasminogen activator (Alkjaersig, Fletcher, and Sherry, 1959).

WHOLE BLOOD CLOTTING TIME After the early acceleration, clotting time lengthened and often became slightly or moderately prolonged. The longest $\frac{\bar{c}}{\bar{c}}$ time was $21 \frac{1}{2}$ minutes (case 3, Fig. 8). Prolonged $\vec{\odot}$ clotting developed in seven out of nine patients with severe injuries compared with three out of eighto with moderate trauma. Figure 8 shows a good $\vec{\circ}$ example: after early acceleration clotting progres-

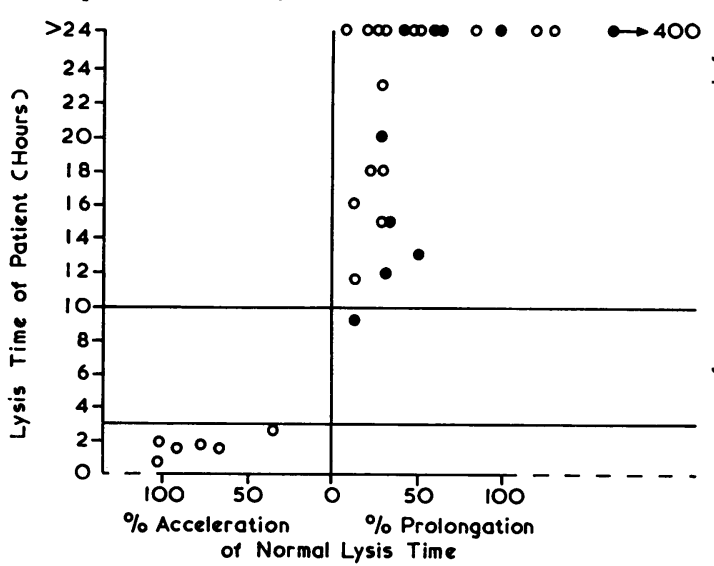

FIG. 7. The results in 12 patients of adding $10 \%$ (open circle) or $20 \%$ (closed circle) of the patient's blood on the $\frac{\mathrm{O}}{\mathrm{O}}$ lysis time of normal samples compared with the lysis time $\varrho$ of the patient's blood. Blood with an accelerated lysis time $\overrightarrow{\overrightarrow{0}}$ accelerates the lysis time of normal blood beyond the 3 dilution effect and blood with a prolonged lysis time $\supset$ prolongs the lysis time of normal blood.

sively slowed during the next three days and waso abnormally slow from the fourth to the fourteenth day or longer. Other cases are shown in Fig. 9:3. clotting was prolonged or near the upper limit for a week or two (cases $4,5,26$ ) or had one or two abnormal peaks (cases 9 and 38).

By adding the antiheparin agent hexadimethrine bromide (polybrene) to the test blood endogenouso heparin was tested for in three cases but clotting times were not shortened. Accelerated clotting waso found in three severely injured, including case $14 \mathrm{~N}$ (Fig. 9), and was probably related to continuedN internal bleeding. In another (case 10, Fig. 2) it also continued from the early period and was eight and three quarter minutes before death at one and a halfe days after injury; the lungs were bleeding from acs rupture and badly contused.

PROTHROMBIN CONSUMPTION Increased consump-o tion indices during the first few hours were found in three out of seven cases (maxima $32 \%$ to $49 \%$ ). In case 5 the index rose to $40 \%$ during the first three or four hours and fell to normal within the next hour 

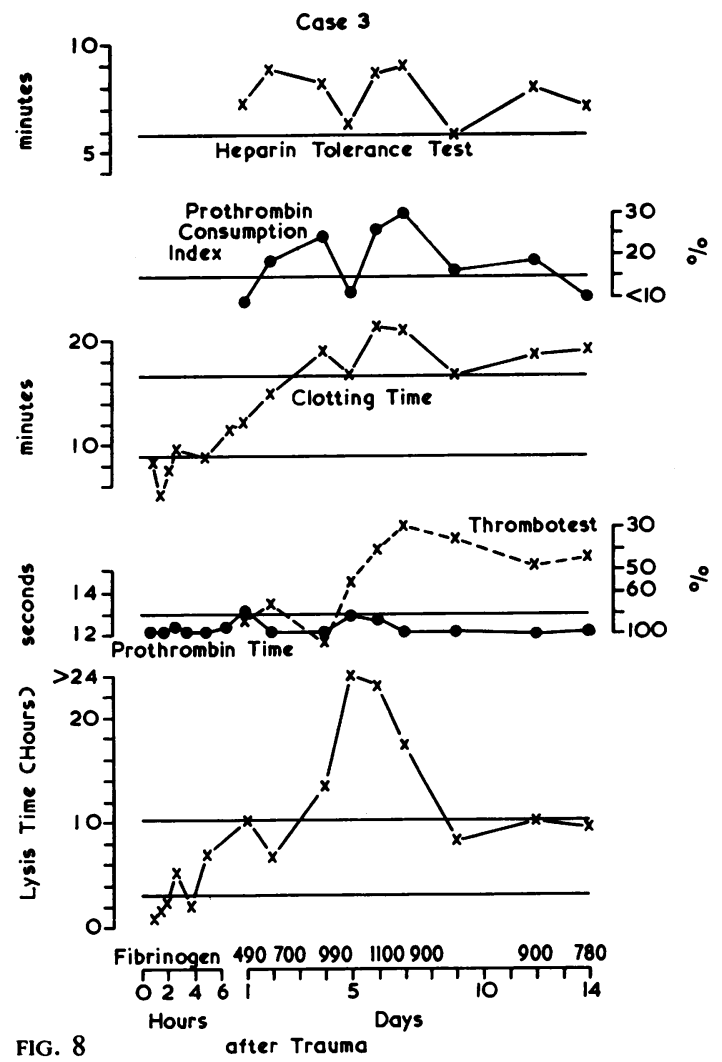

FIG. 8. The early acceleration of clotting time in case 3 is succeeded by a progressive prolongation which becomes abnormal by the third or fourth day after injury and remains prolonged for more than a week. Note the association of prolonged clotting time with an increased prothrombin consumption index (residual serum prothrombin), reduced Thrombotest activity, and the parallel changes in the heparin tolerance test (heparin-retarded clotting time). Prolongation of fibrinolysis time after the early acceleration is delayed in this patient until the fourth day after injury.

During the first week or 10 days indices greater than $15 \%$ were common: the values ranged from $17 \%$ to $>100 \%$ but were mostly $20 \%$ to $40 \%$. Several cases showed a clear association of high indices with prolonged clotting such as case 3 (Fig. 8) and cases 5 and 26 (Fig. 9). Abnormal prothrombin consumption was sometimes associated with normal clotting time (case 9) and occasionally with shortened clotting time (case 14). The overall picture was increased residual prothrombin associated with normal or prolonged clotting time, but unlike haemophilia it was a temporary phenomenon and did not seem to have clinical significance.

PLATElets A fall began during the first few hours after trauma and continued for one to three days when the count was lowest; then the count rose steadily to a thrombocytosis during the next one to three weeks (Fig. 10). Figure 1 shows the fall from 190,000 to 118,000 from two and a half to six hours after injury. Some correlation was found between

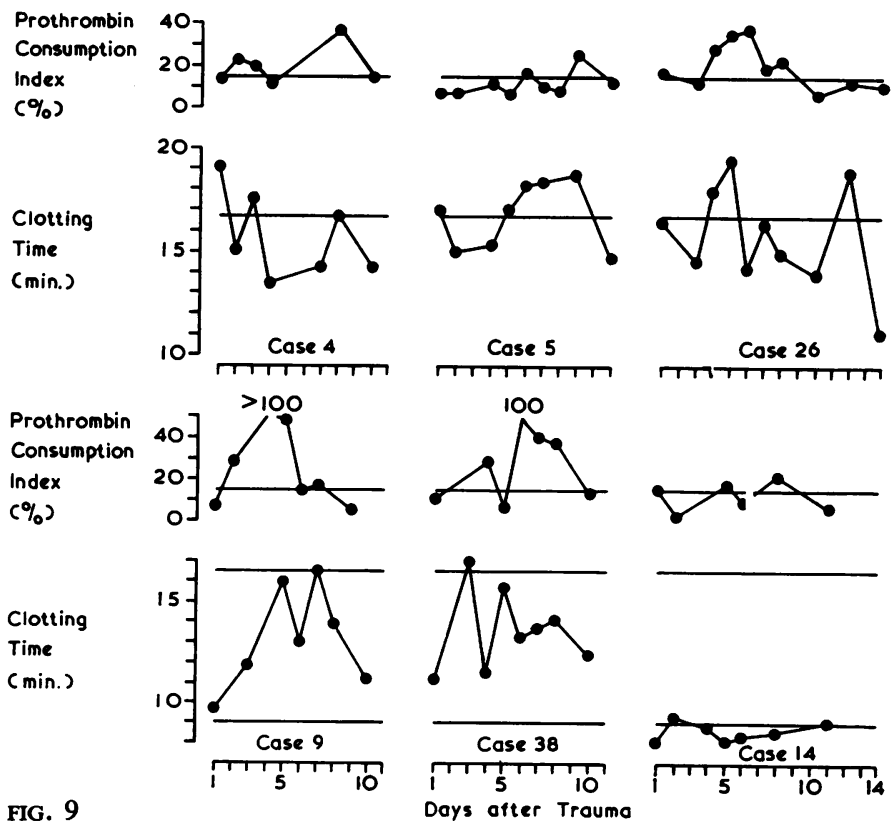

FIG. 9. Clotting times and prothrombin consumption indices in six patients. 
severe injury and the greatest percentage fall at this time although the lowest count during this early period was 95,000 in a subject with only a fractured patella (case 20). Low counts during the first few days were also associated with severe trauma: values less than 150,000 per c.mm. were found in 10 out of 12 with severe injuries and in five out of 14 with moderate injuries; and below 100,000 per c.mm. in three severely and one moderately injured. The lowest counts were 29,000 in case 27 (day after injury) and 76,000 in case 1 (third day).

Subsequent maxima ranged from 241,000 to 1 million per c.mm. among 19 cases studied well enough and were greater than 0.5 million in nine subjects. The highest counts were 715,000 (case 4, sixteenth day), 900,000 (case 5, thirteenth day) and 1 million per c.mm. (case 1, tenth day). Peaks over 0.5 million in six out of 10 with severe trauma and in three out of nine with moderate trauma suggested some association between high counts and severe injury. Values were greatest between the fifth and tenth days in six patients, the eleventh to fifteenth day in another six, and between the sixteenth and twentieth days in seven subjects. Thereafter the counts declined but two peaks occurred in four subjects.
MECHANISM OF TROMBOCYTOPENIA The platelets $\stackrel{0}{\overrightarrow{0}}$

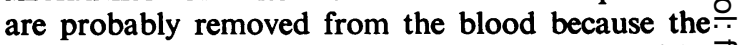
number which disappear can rarely be explained by $\vec{F}$ blood loss or transfusion of platelet-poor blood $\frac{+}{-}$ Thrombosis in vivo may contribute or be responsible:등 platelet and other thrombi were seen histologically in $\overline{\bar{s}}$ the lungs of cases 24 and 25 (and in other injured $\overrightarrow{\widetilde{\sigma}}$

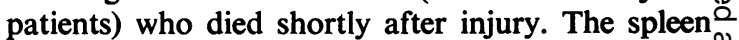
may also be involved since prior splenectomy in bledes rabbits prevented the fall in platelets (Turpini and $\vec{\circ}$ Stefanini, 1959). Since the spleen has a role in re- $\overrightarrow{-}$ moving eosinophils (Sevitt, 1955) and since post- $\omega$ traumatic eosinopenia is due to adrenocortical hyperactivity, the fall in eosinophils and platelets? during the first few hours after injury were compared $\vec{v}$ be serial counts in five subjects. The timing of the fall.in both counts was more or less simultaneous which ${ }_{\circ}$ is consistent with the view that platelet removal may? also be mediated through the adrenal cortex. This ${ }_{C}$ confirms the findings of Warren, Lauridsen, and Belko (1953) and Pepper and Lindsay (1960).

PLASMA FIBRINOGEN Although changes were not $\overrightarrow{0}$ significant during the first few hours (Fig. 4) an increase was present by 24 hours after injury and the fibrinogen level continued to rise for days to reacho

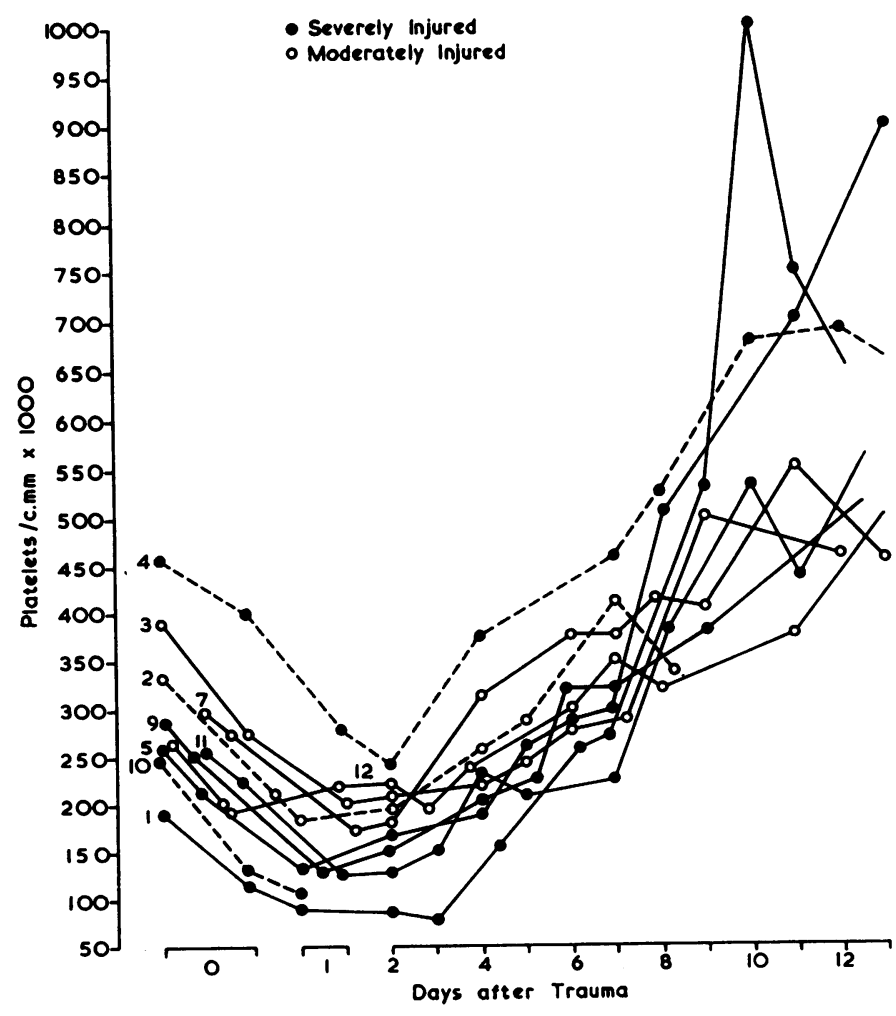

FIG. 10. Platelet counts in 10 patients Numerals refer to case numbers. 
a plateau maximum during the following week (Fig. 11). The rise during the first 24 hours was more than $150 \%$ in five subjects, $100 \%$ to $150 \%$ in three, and $50 \%$ to $100 \%$ in nine. It ranged from $28 \%$ in a patient with a bruised chest and abdomen to $180 \%$ in a subject with multiple injuries. In the burned patient the level was already $510 \mathrm{mg}$. per $100 \mathrm{ml}$. at seven hours after the accident. Figure 11 shows the changes in severely and moderately injured subjects. Fibrinogen increased even when the injuries were relatively minor: it rose from 250 to $720 \mathrm{mg}$. per 100 $\mathrm{ml}$. in a man with only a fractured patella (case 20) and from 290 to $740 \mathrm{mg}$. on the fifth day in a man with a minor head injury (case 30 ). Lysis time, platelet level, and prothrombin time remained normal in them but the clotting time became slightly prolonged. The overall maximum values varied from 660 to $1,200 \mathrm{mg}$. per $100 \mathrm{ml}$. compared with 110 to $500 \mathrm{mg}$. on the day of injury and 300 to $810 \mathrm{mg}$. on the next day. Compared with moderate injury, severe trauma was associated with a prolonged duration of a high fibrinogen level especially among those remaining ill or undergoing a stormy convalescence. For example, in case 11 the fibrinogen level was 600 to $800 \mathrm{mg}$. per $100 \mathrm{ml}$. from the second day until death 15 weeks later; she remained unconscious after a head injury.

Overproduction of fibrinogen by the liver seems to be a common response to various illnesses (Foster and Whipple, 1922; Ham and Curtis, 1938 ) and its association with a fall in prothrombin and other clotting factors after injury is an interesting paradox.

HEPARIN-RETARDED CLOTTING TIME Transient shortening to between three and three quarters and five and three quarter minutes was found in seven of nine patients tested serially during the first few hours after injury but no correlation with clotting time or lysis time was found. In case 24 the result was very prolonged ( $>28$ minutes); prothrombin time was also very prolonged ( $>150$ seconds) and lysis time very quick ( $<$ three quarters of an hour). The patient who had a severe head injury and a fractured ankle was tested at three hours after injury and died half an hour later. Endogenous heparin may have been circultaing.

Later, the test became accelerated in 16 out of

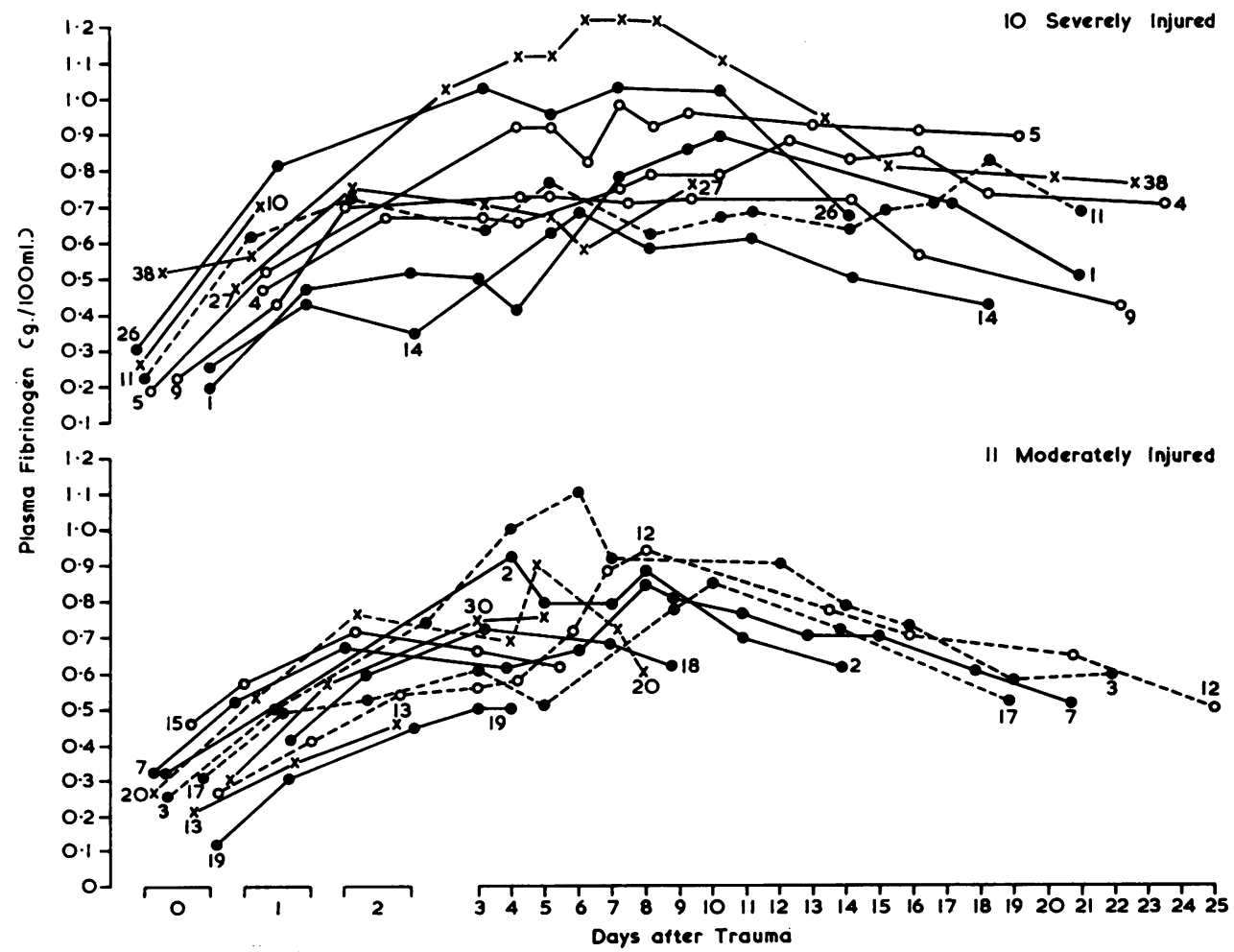

FIG. 11. Plasma fibrinogen concentration in 10 severely and 11 moderately injured subjects. Numerals refer to case numbers. 
22 patients tested serially, in eight during the first five days and in eight subsequently. Plasma antiheparin activity or a reduced heparin cofactor is indicated. There was an interesting association of an early flattening of the rise in plasma fibrinogen in six of the subjects with tests accelerated during the first five days. Deep vein thrombosis is possibly responsible for this and for other accelerated tests.

PROTHROMBIN TIME AND THROMBOTEST Prothrombin times of 14 to 16 seconds and/or low Thrombotest values ( $26 \%$ to $70 \%$ of normal) were frequent during the first few hours after injury (Figs. 1 and 12). Sometimes both tests were abnormal but normal results with one and abnormal results with the other were not infrequent.

Prothrombin times between 14 and 16 seconds were also found in 12 of 23 patients during the first two or three weeks. Prolongation was more common during the first few days and then prothrombin time often returned to normal (Fig. 12, cases 4, 7, 18, and 20). Sometimes a second prolonged phase occurred during the following week (cases 1 and 26) or later (case 4). Thrombotest was usually abnormal for two weeks, sometimes for three or even four weeks or longer after injury: values less than $50 \%$ were found in 15 patients and at or below $30 \%$ in nine. The lowest value was $16 \%$ in case 17 on the second day after injury. Thrombotest values sometimes paralleled prothrombin times in a general way but were often abnormal when the latter was normal, as in case 3 (Fig. 8). Thrombotest fell to between $30 \%$ and $50 \%$ from the fourth day but prothrombin time remained normal. Some subjects showed a tendency to phasic variation as in cases 4 and 7 (Fig. 12).

The results possibly explain the relative sensitivity of many injured patients to oral anticoagulant drugs.

PLASMA PROTHROMBIN The results by the area technique and the two-stage method in case 4 are shown in Figure 13. They were similar except on the day of injury. By the former method there was a fall from normal $(84 \%)$ on the day of injury to $60 \%$ next day and to between $35 \%$ and $50 \%$ during the following week; then there was an interrupted rise. Prothrombin showed some correlation with prolongation of the prothrombin time but not necessarily. Area assays or two-stage tests in 10 other subjects confirmed that prothrombin is reduced to between $30 \%$ and $50 \%$ for one to three weeks after injury.

FACTORS VII AND $x$ Deficiencies were tested for by correction tests. Figure 13 shows that addition of only $2 \%$ of normal serum to the plasma of case 4 reduced the prothrombin time to normal or near

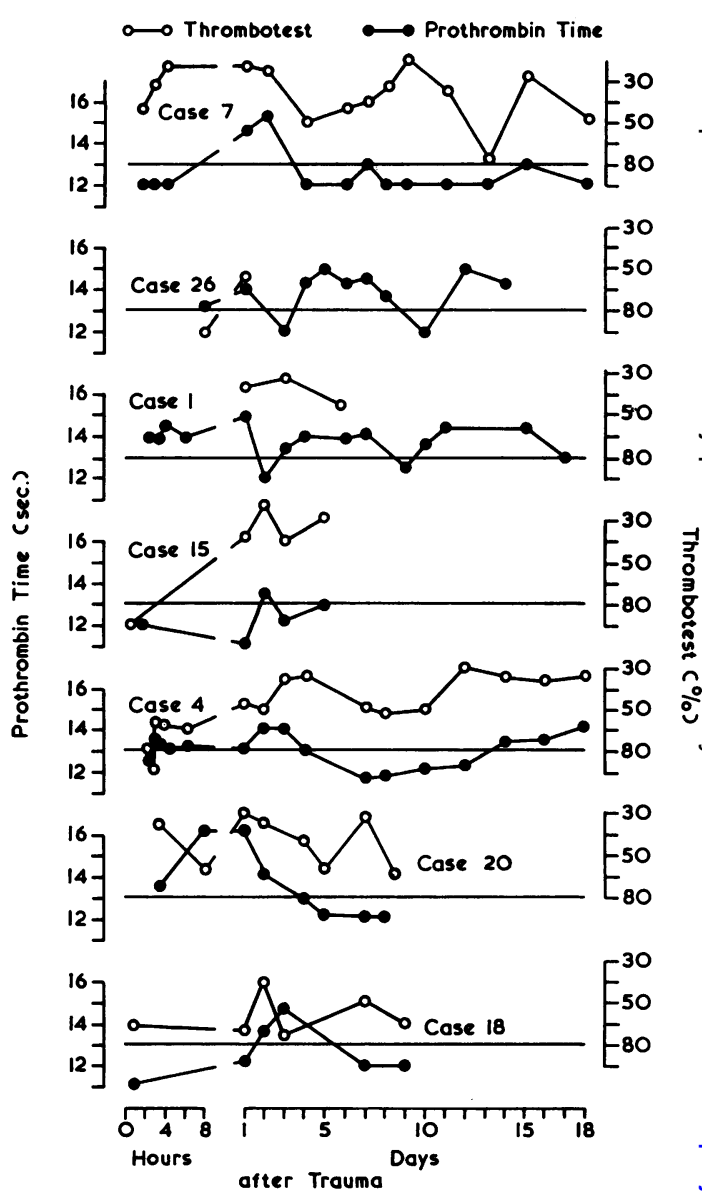

FIG. 12. Serial prothrombin times (solid circles) and Thrombotests (open circles) in seven subjects. Thrombotes? per cent is plotted comparable with prothrombin tim according to the saline dilution calibration curve of the latter.

normal. Serum had a better correcting effect than $10 \%$ normal plasma. This indicates a deficiency in. factors VII or X or both. Other observations support this and Table I shows some of the findings in? case 1 . Plasma adsorbed by $\mathrm{Al}(\mathrm{OH})_{3}$ (rich in factor $\mathrm{V} / \mathrm{S}$ had no correcting effect after the first day (Table I columns 1 (vide infra) ). The prothrombin times of plasmas from patients under phenindione therapyo (not tabulated) and of $\mathrm{Al}(\mathrm{OH})_{3}$ plasmas were्ष shortened by the patient's plasma but normal? plasma had a better correcting effect, except duringo the first two to three hours (Table I, columns 2) when factor $\mathrm{V}$ deficiency may have been largel $\mathbb{S}_{\infty}^{\infty}$ responsible. Tests on other patients from the dasp after injury gave similar results. Using Russell's viper venom as a thromboplastin, the prothrombin 

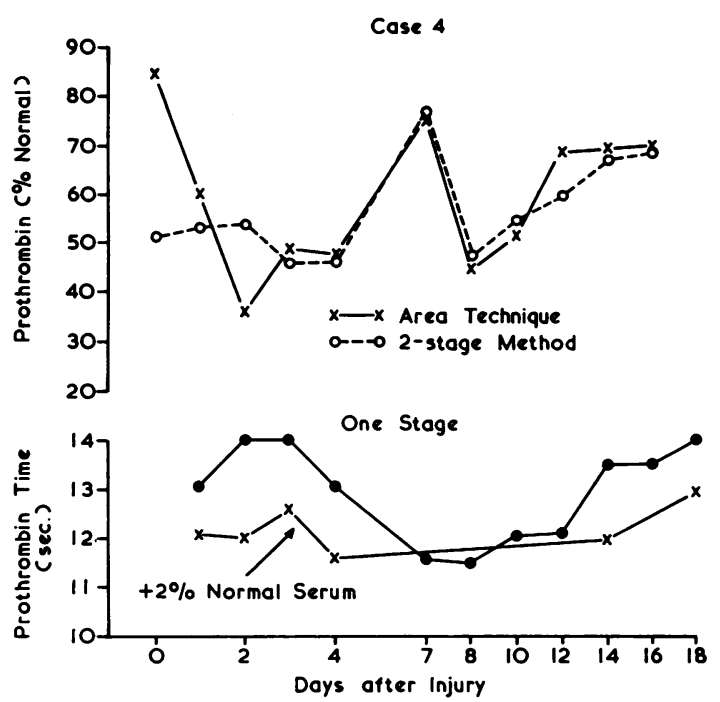

FIG. 13. The fall in plasma prothrombin after injury in case 4 compared with the one-stage prothrombin time.

times of normal plasma and of plasma from case 4 were the same which suggested that factor $X$ was not significantly deficient.

FACTOR $V$ Tests were suggestive of factor $\mathbf{V}$ deficiency on the day of injury but not subsequently (Table I). The patient's prothrombin time was slightly shortened by $\mathrm{Al}(\mathrm{OH})_{3}$-plasma on four out of five occasions during the first few hours (columns 1) but not on the subsequent days. The patient's plasma during the first few hours corrected the prothrombin time of stored plasma (factor V-deficient) less well than did normal plasna (columns 3 ) but later was equally or more capable than normal plasma of correcting the deficit in stored plasma. During the days following the transfusion of 4 litres of blood between three and 24 hours after injury, the patient's prothrombin time was not reduced by $\mathrm{Al}(\mathrm{OH})_{3}$ plasma which suggests that transfusion with stored blood played little part in the early reduction of factor V. This contrasts with the findings of Scott and Crosby (1954) but their patients received massive transfusions. Similar results were found in case 4 after $\mathbf{1 . 5}$ litres of stored blood on the day of injury.

\section{DISCUSSION}

The first few hours after trauma are dominated by speeded clotting and fibrinolysis, the latter beginning very soon after injury and often before quickened clotting was detected. They were accompanied by other changes, rarely exceeded a few hours, and were often followed by a rebound state. These findings agree substantially with those of Bergentz and Nilsson (1961) in dogs with fractured femurs and with most of the coagulation changes found by Scott and Crosby (1954) in battle casualties, by Warren et al. (1950) after surgical operations, and by Miller, Willson, and Eliot (1959) in the dog after trauma.

Our patients had a wide variety of accidental injuries and various operations or orthopaedic manoeuvres were often done on the day of injury or subsequently. Location of injury did not seem to influence acceleration of clotting or lysis; but the severity affected the duration of accelerated lysis and possibly the likelihood of subsequent prolongation. Hypotension was not necessarily involved, and blood clotting or fibrinolytic changes could not be related to changes in blood pressure. Although the degree

TABLE I

CORRECTION TESTS ON DEFICIENCY OF FACTORS $V$ AND VII AND/OR X IN CASE $1^{1}$

\begin{tabular}{|c|c|c|c|c|c|c|c|c|c|c|c|}
\hline \multirow{3}{*}{$\begin{array}{l}\text { Time } \\
\text { after } \\
\text { Injury }\end{array}$} & \multicolumn{10}{|c|}{ One-stage Prothrombin Time (sec.) } & \multirow{3}{*}{$\begin{array}{l}\text { Trans- } \\
\text { fusion of } \\
\text { Stored } \\
\text { Blood }(l .)\end{array}$} \\
\hline & \multicolumn{4}{|c|}{1 Patient's Plasma } & \multicolumn{3}{|c|}{$2 \mathrm{Al}(\mathrm{OH})_{3}-\mathrm{Plasma}$} & \multicolumn{3}{|c|}{3 Stored Plasma } & \\
\hline & $\begin{array}{l}\text { Un- } \\
\text { corrected }\end{array}$ & $\begin{array}{l}+10 \% \\
\text { Normal } \\
\text { Plasma }\end{array}$ & $\begin{array}{l}+2 \% \\
\text { Normal } \\
\text { Serum }\end{array}$ & $\begin{array}{l}+10 \% \\
\text { Al( }(\mathrm{OH})_{3-} \\
\text { Plasma }\end{array}$ & $\begin{array}{l}\text { Un- } \\
\text { corrected }\end{array}$ & $\begin{array}{l}+20 \% \\
\text { Normal } \\
\text { Plasma }\end{array}$ & $\begin{array}{l}+20 \% \\
\text { Patient's } \\
\text { Plasma }\end{array}$ & $\begin{array}{l}\text { Un- } \\
\text { corrected }\end{array}$ & $\begin{array}{l}+10 \% \\
\text { Normal } \\
\text { Plasma }\end{array}$ & $\begin{array}{l}+10 \% \\
\text { Patient's } \\
\text { Plasma }\end{array}$ & \\
\hline $\begin{array}{l}2 \frac{1}{2} \mathrm{hr} . \\
2 \frac{8}{\mathrm{hr}} \\
3 \frac{1}{2} \mathrm{hr} . \\
4 \mathrm{hr} . \\
6 \mathrm{hr} .\end{array}$ & $\begin{array}{l}14 \\
14 \cdot 5 \\
14 \\
14 \cdot 5 \\
14\end{array}$ & $\begin{array}{l}13 \\
13 \\
13 \\
12 \cdot 5 \\
12 \cdot 5\end{array}$ & $\begin{array}{l}\overline{14} \\
\overline{14} \\
13\end{array}$ & $\begin{array}{l}13 \cdot 5 \\
14 \\
15 \\
14 \\
13\end{array}$ & $\begin{array}{l}130 \\
130 \\
130 \\
130 \\
130\end{array}$ & $\begin{array}{l}20 \\
20 \\
20 \\
20 \\
20\end{array}$ & $\begin{array}{l}20 \\
20 \\
24 \\
21 \\
28\end{array}$ & $\begin{array}{l}16 \\
16 \\
16 \\
16 \\
16\end{array}$ & $\begin{array}{l}13 \\
13 \\
13 \\
13 \\
13\end{array}$ & $\begin{array}{l}14 \cdot 5 \\
14 \cdot 5 \\
14 \\
14 \\
14 \cdot 5\end{array}$ & $\uparrow 1.5$ \\
\hline $\begin{array}{l}1 \text { day } \\
3 \text { days } \\
6 \text { days }\end{array}$ & $\begin{array}{l}15 \\
14 \\
14\end{array}$ & $\begin{array}{l}13 \cdot 5 \\
13 \\
12 \cdot 5\end{array}$ & $\begin{array}{l}12 \cdot 5 \\
12 \cdot 5^{2} \\
13\end{array}$ & $\begin{array}{l}15 \\
14 \\
15\end{array}$ & $\begin{array}{l}130 \\
9 \frac{1}{4} \mathrm{~min} . \\
180\end{array}$ & $\begin{array}{l}20 \\
20 \\
19\end{array}$ & $\begin{array}{l}24 \\
22 \\
21\end{array}$ & $\begin{array}{l}16 \\
21 \\
17\end{array}$ & $\begin{array}{l}13 \\
16 \cdot 5 \\
15\end{array}$ & $\begin{array}{l}15 \\
16 \cdot 5 \\
13 \cdot 5\end{array}$ & $\downarrow 2 \cdot 5$ \\
\hline
\end{tabular}

Notes: Serum is rich in factors VII, $\mathbf{X}$; deficient in prothrombin, factor $\mathrm{V} . \quad \mathbf{A l}(\mathrm{OH})_{3}$-plasma (normal plasma adsorbed by aluminium hydroxide) is rich in factor $\mathrm{V}$ and deficient in factors VII, $\mathrm{X}$, and prothrombin. Stored plasma is poor in factor V.

${ }^{1}$ All tests were done on day of venepuncture except the first five which were done next day.

${ }^{2}$ Prothrombin time after adding $10 \%$ normal serum heated to $56^{\circ}$ for 20 min. was 15 sec. (compare Scott and Crosby, 1954 ). 
of haemorrhage is important in accelerating clotting (Gray and Lunt, 1914), tissue damage may also be important, but this was difficult to determine since they were so commonly associated in our patients. When blood loss was considerable the transition from quickening to slowing of lysis and sometimes of clotting sometimes took place during transfusion (Fig. 1), but similar time relations were observed in others requiring little or no transfusion. Estimations of blood volume by the ${ }^{51} \mathrm{Cr}$ technique in five severely injured subjects during the shock phase showed that lysis time can become prolonged when the blood volume is still reduced. Thus, in case 1 (Fig. 1) the blood volume deficit was 1 litre at four and a half hours after injury just when lysis and coagulation times were beginning to lengthen. Quick clotting may continue for a time, as in case 10 who was receiving considerable transfusion for severe haemorrhage from a chest injury: at 26 hours his blood volume deficit was 0.5 litre clotting was still quick but fibrinolysis was very prolonged (Fig. 2).

Lysis time and clotting were also accelerated in patients with injuries unaccompanied by significant blood loss. Pain, fear, emotional excitement, found by Cannon and Mendenhall (1914) to accelerate clotting in cats, may be implicated. Consciousness is not essential since the patients included a few unconscious soon after a head injury. Perhaps stimuli which cause pain in the conscious state are still capable of quickening lysis and clotting time in the unconscious state.

Our results strongly suggest that acceleration of clot lysis is the result of a great excess of plasminogen activator. Plasma fibrinogen was not significantly affected at this time and there was no evidence of a fibrinogenolytic state in vivo. The findings indicate that the activity observed by clot lysis tests is due to activation to plasmin of plasminogen (bound by the fibrin of the clot) by a chemical activator in the plasma and supports the conclusions of others (Sherry, Lindemeyer, Fletcher, and Alkjaersig, 1959). The lytic activity is inactive in the circulation due no doubt to natural inhibitors, but could come into play if and when intravascular clotting took place. The clot-lysis agents and products are evidently harmless or labile because incoagulable cadaver blood has been successfully transfused for many years in Moscow.

To account for acceleration of clotting the entry of a thromboplastin from injured tissue, especially bone marrow, seems plausible but could not explain the acceleration from haemorrhage without trauma. Nevertheless thromboplastin activity after rapid bleeding is indicated by the work of Shafiroff, Doubilet, Siffert, and CoTui (1943) who found that severe bleeding reduced the prolonged clotting time after intravenous peptone, protamine, an heparin; and by the rapid development of powerfui clot-accelerating properties by plasma and serur $\overrightarrow{\bar{z}}$ in severely bled rabbits (Turpini and Stefanini, 19595

Quickening of fibrinolysis and clotting may be triggered off by the same mechanism. Suffice it to sa here that adrenaline is likely to be implicated a least as a trigger mechanism (Cannon and Grayo 1914; Biggs, Macfarlane, and Pilling, 1947; Sherr's et al., 1959) and the possibility that both are sẹb in motion by activation of the Hageman factor needs to be explored. The clue provided by McClintock and Magers (1926) also needs invest gation; they found that clotting in severely bled dog did not quicken after prior splenectomy.

The onset of prolonged fibrinolysis is abrupt and our observations suggest that it is probably due to flooding of the circulation by an inhibitor. This is possibly an anti-activator. Prolonged clotting develops more slowly and seems related to a cont plex and possibly variable deficiency in clotting factors. The limited data are against heparin to account for the slow clotting although the proximit $\overrightarrow{x b}$ of mast cells to vessels makes the hypothesis tempe ing. In one patient (case 24) there was a possible anticoagulant effect but that was in the early phase and soon before death. Heparin may only be released in some very badly injured patients and this requires further investigation. In animal expero ments the evidence is conflicting. In severely blef dogs no evidence of heparin release was found bo Smith, Grace, and Hussey (1958) but a lipaemic clearing effect in the plasma and other evidence of endogenous heparin during the early phase was reported by Hardaway et al. (1962).

The antiheparin activity of the plasma was ofte increased in our patients similar to the resistance tô. heparin after operation reported by others (Waugh and Ruddick, 1944; Warren et al., 1950; Polle 1954; Gormsen and Haxholdt, 1961). Prothrombia and factor VII also fell and there was often a rise in the residual serum prothrombin consistent with deficiency of a factor or factors involved in the ear $\overline{\bar{V}}$ stage of thromboplastin generation. Some workeß report a fall in factor V (Scott and Crosby, 1954 Turpini and Stefanini, 1959; Bergentz and Nilssotu 1961) and our evidence of its deficiency was restricted to the first few hours after injury. The early de ficiencies in prothrombin and in factors $\mathrm{V}$ and $\mathrm{V}$ might be due to consumption by coagulation in vive which would also help to explain the early fall in platelets. Bergentz and Nilsson (1961) reported that previous heparinization prevented the early fall $\overrightarrow{\text { A }}$ antihaemophilic globulin (VIII) and factor V afte fractures in dogs. The normal values of factors and VII and the increase in VIII, IX, and X found 
by Davidson and Tomlin (1963) at 10 to 20 days after fracture do not preclude decreased levels before this time.

Liver function may also be involved. A phase of liver dysfunction is common after injury and burns, and though ordinarily not clinically recognisable, it is enhanced by anaesthesia and operation and may lead to jaundice after compatible blood transfusion (Sevitt, 1958). It could help explain the fall in prothrombin and factors V and VII, particularly since the half-life of the latter is only a matter of hours.

HOMEOSTASIS AND ITS POSSIBLE BREAKDOWN The early acceleration of clotting seems an emergency mechanism against continued haemorrhage after wounding, helping local haemostasis and supplementing the vasoconstrictive effect of adrenaline on torn vessels whereas the speeding of clot lysis at this time is presumably a guard against thrombosis in vivo elsewhere. The subsequent inhibition of clot lysis may also be protective, neutralizing the flood of activator in the circulation and guarding against the breakdown of the mechanism which prevents fibrinogenolysis and other proteolytic effects of plasmin in the blood. Its prolonged duration and its appearance after little or no accelerated lysis suggests an excessive rebound; but it may have a greater significance since prolonged fibrinolysis is common in various medical and surgical conditions (Guest, Daly, Ware, and Seegers, 1948).

A breakdown in this mechanism has been postulated to explain the 'irreversible' shock found in dogs bled to severe hypotension and unable to respond to re-infusion of the blood. Thrombi appear in the lungs, splanchnic circulation, and elsewhere and are said to prohibit a favourable response to re-infusion (Crowell and Read, 1955; Turpini and Stefanini, 1959; Hardaway, 1962). Experimental studies in vivo by microcinematography have confirmed microthrombi in the circulation during haemorrhage and other kinds of shock (Robb, 1963). Hardaway believes that local thrombosis produces the haemorrhagic necrosis of bowel found in the dog and that this permits the entry of bacteria and bacterial toxins. Heparinization before bleeding is said to permit survival but others have been unable to obtain clearcut results (Smith, Grace, and Hussey, 1958). Infusion of 'fibrinolysin' after haemorrhage was also claimed to permit a life-saving response to the return of blood. (Hardaway and Burns, 1963). Capillary thrombi were said to be lysed and the blocked circulation restored. Robb (1963) confirmed that micro-thrombi in the circulation after experimental shock are prevented or lessened by combined 'fibrinogen' and heparin therapy.

Histological examination showed some small thrombi in the lungs of cases 24 and 25 who died within a few hours of injury. Case 24 was the patient with evidence of a circulatory anticoagulant. Clotting was accelerated in case 25 and fibrinolysis was very quick in both; pulmonary thrombi have also been found in other subjects dying within a day or so of injury or burns and a systematic necropsy study of early micro-thrombosis after trauma is ovbiously required.

A research grant from the Birmingham Regional Hospital Board is gratefully acknowledged. We thank our clinical colleagues, technical and nursing staff for their cooperation, the Photographic Department for help in the preparation of the figures, and Dr. A. Darragh of Leo Laboratories, Dublin, for the gift of urokinase.

\section{REFERENCES}

Alkjaersig, N., Fletcher, A. P., and Sherry, S. (1959). J. biol. Chem., 234,832 .

Bergentz, S-E., and Nilsson, I. M. (1961). Acta chir. scand., 122, 21.

Biggs, R., and Douglas, A. S. (1953). J. clin. Path., 6, 23.

, and Macfarlane, R. G. (1953). Human Blood Coagulation. Blackwell, Oxford.

-

Brecher, G., and Cronkite, E. P. (1950). J. appl. Physiol., 3, 365.

Cannon, W. B., and Gray, H. (1914). Amer. J. Physiol., 34, 232.

_- and Mendenhall, W. L. (1914). Ibid., 34, 225, 243, 251.

Cliffton, E. E. (1952). J. Lab. clin. Med., 39, 105.

Crowell, J. W., and Read, W. L. (1955). Amer. J. Physiol., 183, 565.

Davidson, E., and Tomlin, S. (1963). J. clin. Path., 16, 112.

Fearnley, G. R., Balmforth, G., and Fearnley, E. (1957). Ibid., 16, 645. -, Revill, R., and Tweed, J. M. (1952). Clin. Sci., 11, 309.

Foster, D. P., and Whipple, G. H. (1922). Amer. J. Physiol., 58, 393 and 407

Gormsen, J., and Haxholdt, B. Fl. (1961). Acta chir. scand., 121, 377.

Gray, H., and Lunt, L. K. (1914). Amer. J. Physiol., 34, 332.

Guest, M. M., Daly, B. M., Ware, A. G., and Seegers, W. H. (1948). J. clin. Invest., 27, 793.

Ham, T. H., and Curtis, F. C. (1938). Medicine (Baltimore), 17, 413. Hardaway, R. M. (1962). Ann. Surg., 155, 325.

- Brune, W. H., Geever, E. F., Burns, J. W., and Mock, H. P. (1962). Ibid., 155, 241.

-, and Burns, J. W. (1963). Ibid., 157, 305.

Hewson, W. (1846). The Works of William Hewson, F.R.S., edited by G. Gulliver, p. 46. Sydenham Society, London.

McClintock, J. T., and Magers, E. (1926). Proc. Soc. exp. Biol. (N.Y.), 24, 203.

Macfarlane, R. G., and Biggs, R. (1946). Lancet, 2, 862.

Miller, W. R., Willson, J. T., and Eliot, T. S. (1959). Angiology, 10, 375 .

Mole, R. H. (1948). J. Path. Bact., 60, 413.

Pepper, H., and Lindsay, S. (1960). Surg. Gynec. Obstet., 110, 319.

Poller, L. (1954). Angiology, 5, 21.

Robb, H. J. (1963). Ann. Surg., 158, 685.

Scott, R. Jr., and Crosby, W. H. (1954). Blood, 9, 609.

Sevitt, S. (1955). J. clin. Path., 8, 42.

(1958). Brit. J. Surg., 46, 68.

Shafiroff, B. G. P., Doubilet, H., Siffert, R., and CoTui (1943). Amer. J. Physiol., 138, 753.

Sherry, S., Lindemeyer, R. I., Fletcher, A. P., and Alkjaersig, N. (1959). J. clin. Invest., 38, 810.

Smith, J. J., Grace, R. A., and Hussey, C. V. (1958). Amer. J. Physiol., $193,593$.

Tagnon, H. J., Levenson, S. M., Davidson, C. S., and Taylor, F. H. L. (1946). Amer. J. med. Sci., 211, 88.

Turpini, R., and Stefanini, M. (1959). J. clin. Invest., 38, 53.

Warren, R., Amdur, M. O., Belko, J., and Baker, D. V. (1950). Arch. Surg, 61, 419.

__, Lauridsen, J., and Belko, J. S. (1953). Circulation, 7, 481.

Watson, D. (1961). Brit. med. J., 1, 903.

Waugh, T. R., and Ruddick, D. W. (1944). Canad. med. Ass. J., 50, 547. 